\title{
Visual acuity and the ability of the visually impaired to read medication instructions
}

\author{
S R Drummond, R S Drummond, G N Dutton
}

Br J Ophthalmol 2004;88:1541-1542. doi: 10.1136/bjo.2003.029918

Background/Aims: Although medical information is often communicated in writing, little attention is given as to whether patients can read it-for example, the miniscule writing on the side of the medication bottles (equivalent to $6 / 6$ reduced Snellen). The authors wished to determine a distance visual acuity threshold, using routine assessments of visual acuity (VA), beyond which patients are unable to read their instructions.

Methods: 180 patients, subgrouped according to the best line of Snellen acuity (from 6/9 to 6/60) achieved in either eye were recruited. Subjects were asked to read the printed manufacturer's instructions on the side of the drops box without magnification.

Results: Those subjects with best corrected VA of lower than or equal to $6 / 24$ showed a significantly diminished ability to read the instructions on their eye drops bottles $(p<0.001$ for each comparison). When this group selected a font size of their choosing, the mode for preferred Arial font sizes were 16 for the $6 / 24$ group, 18 for $6 / 36$, and 22 for $6 / 60$.

Conclusion: This study documents the inability of patients with visual impairment to read the instructions on their bottle of eye drops. There is evidence of a distance VA threshold effect such that, although the majority of patients with $6 / 18$ VA are able to read their instructions, patients whose VA is worse cannot. This latter group would benefit from a larger font size, a size of Arial 22 being sufficient. We recommend that all printed information for patients with visual acuities between $6 / 24$ and $6 / 60$ be printed in font Arial 22 or equivalent.

\footnotetext{
A ccording to World Health Organization criteria, the prevalence of visual impairment ranges from $0.1 \%$ to $11.8 \%$ depending on the age group involved. ${ }^{1}$ Although medical information is often communicated in writing, little attention is given as to whether patients can read it. This is particularly true for instructions on eye drop bottles prescribed to the visually impaired. These instructions are routinely written in a miniscule font size (equivalent to $6 / 6$ reduced Snellen) to enable them to fit on the side of the small bottle. Consequently, many visually impaired patients are unable to read the instructions for their treatment. We wished to determine a distance visual acuity (VA) threshold beyond which patients are unable to read their instructions, using routine assessments of VA thus enabling identification of those patients who would benefit from enlarged print.
}

\section{SUBJECTS AND METHODS}

The patient group was randomly selected from those patients attending the Tennent Institute of Ophthalmology, Glasgow. These patients routinely have their spectacle corrected distance VA in both eyes assessed as they enter the department. Best corrected VA for each eye was defined as the smallest line of print on the Snellen distance VA chart which the patient was able to read with a single eye, mistaking no more than one letter. Thirty patients for each of the six levels of best corrected VA between and including 6/9 and 6/60 were collected, recruiting 180 patients in total. In a pilot study, patients with distance VA of 6/6 were uniformly able to read the small print on medication bottles, whereas patients with a distance VA worse than 6/60 were uniformly unable to read the same small print. Therefore these groups were excluded from the study. For each participant, sex and age were noted in addition to their main visual complaints.

Near vision was assessed with the subject in natural light, wearing their usual reading spectacles if appropriate, using near vision Snellen acuity charts and near vision cards held at one third of a metre. All readings were made by the same assessor in the same surroundings. Subjects were then asked to read the instructions on the side of the box of drops without magnification. Subjects were permitted to take the box as near to their face as desired because this is what would happen at home. If reading was possible, the time taken for this to be achieved was noted. A time of greater than 30 seconds was recorded as "reading with difficulty". Finally, the subject chose the size of font with which they could read comfortably from a standard selection. Statistical analysis was performed using Minitab version 13.1. The nonparametric Kruskal-Wallis test was used with Dunn's test to compare each group. A p value of $<0.05$ was considered statistically significant.

\section{RESULTS}

The mean age was 70 years (range 23-100 years). Seventy seven $(43 \%)$ were male and 103 (57\%) female. There were 11 main categories of pathology: glaucoma, $n=45$ (25\%); age related macular degeneration, $\mathrm{n}=34$ (19\%); retinal/macular pathology, $\mathrm{n}=20$ (11\%); cataract, $\mathrm{n}=19$ ( $11 \%)$; diabetic retinopathy, $\mathrm{n}=22(12 \%)$; unspecified, $\mathrm{n}=11(6 \%)$; corneal, $\mathrm{n}=10 \quad(6 \%)$; congenital condition, $\mathrm{n}=6 \quad(3 \%)$; vascular event, $\mathrm{n}=5$ (3\%); optic neuropathy, $\mathrm{n}=4(2 \%)$; and uveitis $\mathrm{n}=4,(2 \%)$.

Our results showed there was a decline in near VA with decline in distance VA measured using both the near VA test types and the Snellen reduced VA chart (fig 1).

When comparing the ability to read the instructions on the medication with distance VA, there was a significant difference between the subgroup of patients with a best distance VA of $6 / 18$, most of whom were able to read the instructions and the subgroup with a best distance VA of 6/24 who were unable to read the instructions (fig 2).

Moreover when these latter groups were able to select the font used the mode for preferred Arial font sizes were 16 for the $6 / 24$ group, 18 for $6 / 36$, and 22 for $6 / 60$.

Abbreviations: VA, visual acuity 


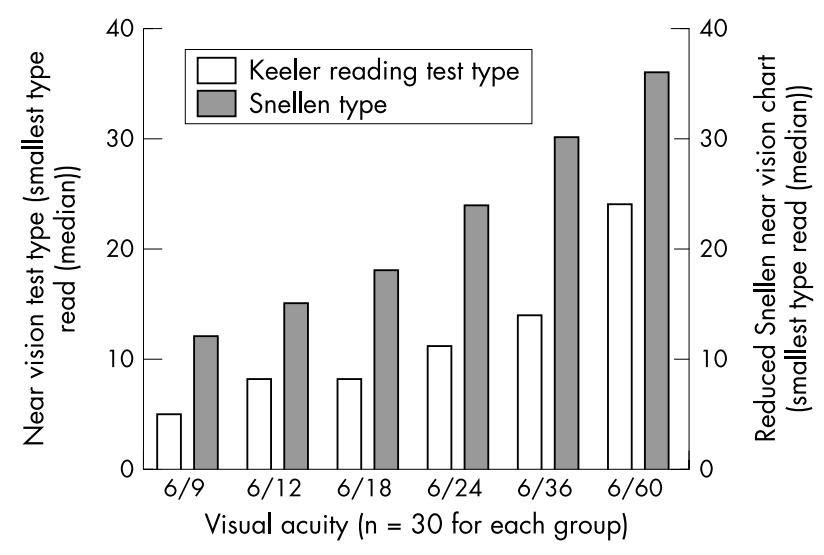

Figure 1 Near visual acuity (VA) (near vision test type and reduced Snellen chart) versus distance VA. Graph documenting the decline in median near visual acuity, assessed using both the near vision test types and the reduced Snellen acuity chart, with a decline in distance VA. There is good correlation between the two methods of assessing near visual acuity $(r=0.95, p=0.004)$.

\section{DISCUSSION}

To our knowledge, this is the first study to document the inability of patients with visual impairment to read the instructions on their bottle of eye drops. Furthermore there is evidence of a distance VA threshold effect such that although the majority of patients with 6/18 VA are able to read their instructions, patients whose VA is worse cannot. As it is common for patients to leave a consultation without remembering what was discussed, ${ }^{2}$ including how to take their medication, this has significant implications in clinical practice as it provides evidence that we need to include enlarged print instructions for patients with a VA of 6/24 or less to enable them to self medicate safely and effectively. This is particularly true for elderly patients for whom hearing impairment and memory problems can compound the difficulties. ${ }^{3}$ Furthermore, in line with the Disability Discrimination Act (1995), ${ }^{4}$ we need to provide a "level playing field" for people who otherwise face obstacles and barriers in their everyday lives. Part 3 of the Act (starting October 2004) requires the provider of a service to take such steps as it is reasonable to enable disabled people to make use of a service, or to facilitate the use by disabled persons of such a service. For a person with visual impairment as described above, the provision of enlarged print would enable the correct usage of medications.

We wished to provide an easy, inexpensive method of assessing whether patients would require enlarged print. Although near VA can be assessed using the near test types and reduced Snellen charts, this requires extra time and personnel. As we have shown and as one would expect, the median near VA decreases as the best distance VA decreases. Therefore, to judge whether a patient will need enlarged print to read their medication instructions, the distance VA can suffice. As all ophthalmology patients have their distance VA assessed as a matter of course, it does not provide an increased workload.

In the provision of enlarged print instructions, we found that the affected group with distance VA of $6 / 24$ to $6 / 60$ was able to read an Arial font size of 22 and thus this size is effective for all affected groups with an acuity of $6 / 60$ or better.

The small number of patients with VA of $6 / 24$ or less who were able to read the small writing tended to be younger patients (age 40 or less), mostly suffering with congenital conditions, whose youth presumably facilitated accommodation.

We would recommend that a copy of instructions, printed in enlarged print of Arial font 22, be distributed with all

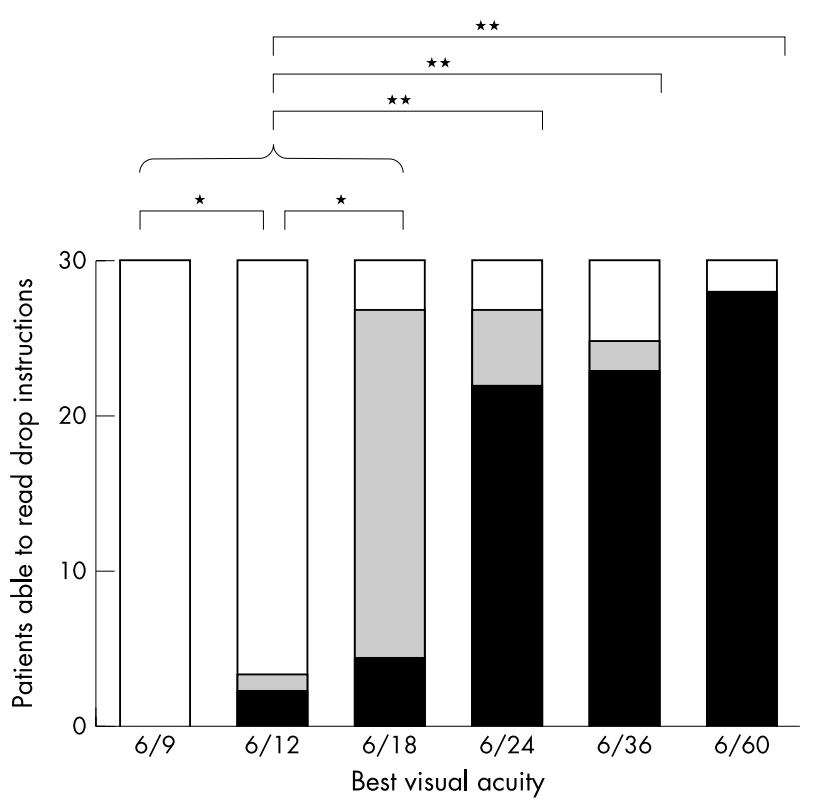

Able to read instructions without difficulty Able to read instructions with difficulty Not able to read instructions

Figure 2 Ability to read medication instructions related to distance Snellen best eye visual acuity (VA). Graph tabulating the sharp difference between the subgroup of patients with a best distance VA of $6 / 18$, most of whom were able to read the instructions, and the subgroup with a best distance VA of $6 / 24$ who were unable to do the same. For statistical analysis the non-parametric Kruskal-Wallis test was used with Dunn's test to compare each group. A p value of $<0.05$ was considered statistically significant. This graph shows that there was no statistical significance between ability to read and "best" VA with those subjects with best visual acuities of $6 / 9,6 / 12$, or $6 / 18$ ( $p>0.05$ in each case). However, when these subjects were grouped and compared with subjects with "best" VAs of $6 / 24,6 / 36$, and $6 / 60$, the latter three groups showed a significantly diminished ability to read the instructions on their eye drop bottles ( $p<0.001$ in each comparison). ${ }^{*} p>0.05$; ${ }^{* *} p<0.001$.

bottles of medication when the patient is found to have a best distance VA of $6 / 24$ or less, particularly if the patient is elderly. This recommendation is probably applicable for all information given to patients with reduced VA.

\section{Authors' affiliations}

S R Drummond, Specialist Registrar in Ophthalmology, Tennent Institute of Ophthalmology, Gartnavel General Hospital, Glasgow, UK R S Drummond, Chest Heart \& Stroke Scotland Research Fellow, British Heart Foundation Glasgow Cardiovascular Research Centre, University of Glasgow Division of Medicine and Cardiovascular Sciences, Glasgow, UK

G N Dutton, Consultant Ophthalmologist, Tennent Institute of Ophthalmology, Gartnavel General Hospital, Glasgow, UK

Correspondence to: Dr S R Drummond, Ophthalmology Department, Tennent Institute of Ophthalmology, Gartnavel General Hospital, Glasgow G12 OYN, UK; suzannah.drummond@virgin.net

Accepted 30 March 2004

\section{REFERENCES}

1 World Health Organization. International Statistical Classification of Diseases and Related Health Problems, Tenth Revision. Vol 1. Geneva, Switzerland: World Health Organization, 1992.

2 Fletcher C. Listening and talking to patients. BMJ 1980;281:994-6.

3 Menon GK, Dutton GN. Writing to our patients. Br J Ophthalmol 1999;83:765.

4 Disability Discrimination Act 1995 (c.50), Part III, (21), 4. 